\title{
La Critique textuelle en rupture avec la grille épistémologique : esquisse d'une analyse des recueils de proverbes gallois du Moyen Âge
}

Textual criticism at odds with the epistemological framework: proposed analysis of medieval Welsh proverb collections

\section{Richard Glyn Roberts}

\section{OpenEdition Journals}

Édition électronique

URL : https://journals.openedition.org//bl/1593

DOI : $10.4000 / \mathrm{lbl} .1593$

ISSN : 2727-9383

Éditeur

Université de Bretagne Occidentale - UBO

\section{Édition imprimée}

Date de publication : 1 novembre 2011

Pagination : 75-85

ISBN : 978-2-901737-92-6

ISSN : $1270-2412$

\section{Référence électronique}

Richard Glyn Roberts, "La Critique textuelle en rupture avec la grille épistémologique : esquisse d'une analyse des recueils de proverbes gallois du Moyen Âge », La Bretagne Linguistique [En ligne], 16 | 2011, mis en ligne le 01 mai 2021, consulté le 22 mai 2021. URL : http://journals.openedition.org//bl/1593 DOI : https://doi.org/10.4000/lbl.1593

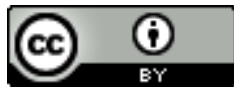

La Bretagne Linguistique est mise à disposition selon les termes de la Licence Creative Commons Attribution 4.0 International. 
Richard Glyn ROBERTS*

\title{
La Critique textuelle en rupture avec la grille épistémologique : esquisse d'une analyse des recueils de proverbes gallois du Moyen Âge
}

\begin{abstract}
A
u cours des années vingt, l'érudit polonais Joseph Morawski a consacré toute une série d'études aux recueils d'anciens proverbes français ${ }^{1}$. Pareillement en Galles, les colosses de l'érudition galloise de l'entre-deux-guerres - Ifor Williams, Henry Lewis, T. H. Parry-Williams - commençaient à éditer les recueils de proverbes gallois du Moyen Âge ainsi que des textes d'intérêt parémiologique tels que le poème didactique Englynion y Clyweit et les traductions galloises de Salomon et Marcolfus ${ }^{2}$. Depuis lors l'étude des
\end{abstract}

* University College Dublin

1. Joseph MoRawSKi, "Les recueils d'anciens proverbes français analysés et classés », Romania 48 (1922), p. 481-558; id., «Locutions et proverbes obscurs», Romania 50, 1924, p. 499-514 ; id., Les diz et proverbes des sages, Paris, PUF, 1924 ; id., Proverbes français antérieurs au XVe siècle, Paris, Champion, 1925 ; id., "Trente-six locutions tirées du ms. 550 de la Bibl. Sainte-Geneviève», Romania 54 (1928), p. 480-484.

2. Ifor Williams et T. H. Parry-Williams, «Englynion y Clyweit», BBCS 3, 1926-1927, p. 4-21 ; Ifor WiLLIAMS, «Hen Ddiarhebion», BBCS 3, 1926-1927, p. 22-31 ; Henry LEWIS, «Salomon et Marcolfus», $B B C S$ 3, 1926-27, p. 161176, 272-285 ; id., «Diarhebion ym Mheniarth 17», BBCS 4, 1927-1929, p. 117 ; id., «Selyf a Marcholffus », BBCS 6, 1931-1933, p. 314-323. 
proverbes en ancien français a été approfondie par leur élucidation dans un cadre comparatif aussi bien que par leur contextualisation, d'une manière systématique, dans la littérature française de l'époque $^{3}$. Par contre, les études parémiologiques galloises n'ont guère progressé et le plus copieux recueil, celui du célèbre Livre Rouge de Hergest, demeure jusqu'à présent inédit ${ }^{4}$.

Les sources pour l'étude des proverbes gallois antérieurs au $\mathrm{XV}^{\mathrm{e}}$ siècle se ramènent essentiellement à quatre recueils, dont deux ne sont conservés que dans un seul manuscrit :

- Peniarth 29 (Le Livre Noir de Chirk). Ms. juridique du XIII siècle, au milieu duquel est inséré un recueil de 88 proverbes copiés par une main légèrement plus récente que celle du texte principal du manuscrit ${ }^{5}$.

- Peniarth 2 (Le Livre Blanc de Rhydderch). Ms. exécuté vers le milieu du XIVe siècle, contenant un bon nombre de textes gallois en prose, parmi lesquels un recueil de 217 proverbes rangés par ordre alphabétique des initiales ${ }^{6}$.

- Jesus College 111 (Le Livre Rouge de Hergest). Ms. copié peu après 1382 par des scribes multiples, contient une vaste anthologie de textes gallois en prose et en vers. Il renferme deux recueils de proverbes d'une ampleur plus imposante, rangés par ordre des lettres initiales, l'un comprenant 615 proverbes, l'autre en contenant 1085. Les deux recueils sont conservés aussi dans Wynnstay 36, qui est un manuscrit contemporain du Livre Rouge. Il subsiste également des versions partielles du recueil le plus grand, dont la plus significative est celle

3. Une étude fondamentale du contexte littéraire a été livrée par E. SCHULZEBUSACKER : Proverbes et expressions proverbiales dans la littérature narrative du Moyen Âge français, Paris, Champion, 1985. L'ampleur de l'aspect comparatif s'est imposé grâce aux travaux de S. SINGER, culminant dans l'édition, par le Kuratorium Singer de l'Académie suisse des sciences humaines et sociales, du monumental Thesaurus Proverbiorum Medii Aevi, Berlin, De Gruyter, 19952000

4. Édition en préparation par l'auteur.

5. Édité par Williams, «Hen Ddiarhebion».

6. Édité par E. Phillimore, «A fragment from Hengwrt MS. No. 202», Y Cymmrodor 7, 1896, p. 89-154. 
conservée dans Peniarth 17, manuscrit qui date de la seconde moitié du XIII ${ }^{\mathrm{e}}$ siècle.

La plupart des proverbes que renferment ces recueils peuvent se classer en quatre groupes principaux selon leur origine :

- Proverbes locaux.

- Axiomes de droit.

- Proverbes qui remontent à la poésie ancienne, c'est-à-dire des citations du Hengerdd.

- Proverbes traduits.

Ce classement implique qu'un proverbe quelconque a un contexte antérieur à son incorporation dans les recueils. C'est un point capital de l'exégèse parémiologique. Car, au-delà de l'analyse purement linguistique des éléments lexicaux et syntaxiques, la pleine signification d'un proverbe demeure opaque et ne se révèle que par son emploi dans un contexte particulier. Pour cette raison, l'élucidation d'un proverbe exige un travail de contextualisation qui fait surgir ce contexte perdu et remet le proverbe dans ses conditions d'usage à l'époque. Pour les trois premiers groupes ci-dessus la contextualisation procède des textes gallois, littéraires ou autres, qui contiennent les proverbes en question. En voici quelques exemples qui illustrent l'approche :

- Hir hun uaelg6n yn egl6ys ros (Le long sommeil de Maelgwn à l'église de Rhos) ${ }^{7}$.

Ce proverbe trouve son origine dans une anecdote qui raconte la mort du roi Maelgwn Gwynedd, un personnage historique qui figure également dans la légende (dans Ystoria Taliesin notamment) et la pseudo-histoire. Selon les Annales Cambriae, il mourut aux environs de 547. Ayant cherché refuge dans l'église de Rhos lors d'une épidémie de «la peste jaune», le roi aurait contracté la maladie en regardant par la porte entrouverte de l'église. Il avait tellement l'air de dormir lorsqu'il disparut que nul n'osait le réveiller. Le proverbe est bien attesté dans la littérature du Moyen Âge et de la Renaissance. Les cywyddwyr, poètes du Moyen Âge tardif, le citent souvent dans leurs élégies composées à la mort d'un mécène parmi la noblesse 
mais l'exemple qui suit est moins typique, donc plus fascinant. Il vient d'un poème de demande dans lequel le poète exige l'octroi d'un lit de la part de quatre femmes nobles. Le poète en question, Lewys Glyn Cothi, détourne la signification usuelle du proverbe puisqu'il ne s'agit plus de mourir comme si on somnolait mais de dormir comme si on était mort. On comprend que le lit qu'il désire est un modèle hyperconfortable :

Ar hwnnw pan fo hirnos

Y gwneir hun Maelgwn yn Rhos ${ }^{8}$.

(Sur celui-là quand la nuit est longue/ On dort comme Maelgwn à Rhos.)

William Salesbury, humaniste du XVI ${ }^{\mathrm{e}}$ siècle et traducteur du Nouveau Testament, en fournit un autre exemple. Salesbury a fait publier, en 1547, le recueil de proverbes compilé par le poète Gruffudd Hiraethog mais l'extrait ci-dessous vient de son herbier médicinal, là où il traite des effets de la belladone :

Ac od yfir pwys pedwar dram peri cyscy hûn Vaelgwn yn eglwys Rôs a wna ${ }^{9}$.

(Et si on boit le poids de quatre drachmes elle [la belladone] fait somnoler le sommeil de Maelgwn à l'église de Rhos.)

- Telittor g6edy hala6c $l 6$ (On paie après un serment souillé) ${ }^{10}$.

L'emploi de la forme telittor, vieille forme impersonnelle du verbe talu (payer) au présent de l'indicatif, suggère qu'il s'agit d'un proverbe fort ancien mais la signification exacte de ce brocard est parfaitement impénétrable hors contexte. C'est vers les codes de lois gallois, que la tradition attribue à l'initiative du roi Hywel Dda, qu'il faut tourner notre regard afin de restituer le proverbe dans sa singularité historique. Il est cité dans le contexte particulier de $l l w g r$ $\hat{y} d$, c'est-à-dire du blé abîmé par un animal ou des animaux :

8. Dafydd Johnston, Gwaith Lewys Glyn Cothi, Caerdydd, Gwasg Prifysgol Cymru, 1995, $\mathrm{n}^{\circ}$ 186, lignes 53-4.

9. Iwan Rhys Edgar, Llysieulyfr Salesbury, Caerdydd, Gwasg Prifysgol Cymru, 1997, p. 135.

10. J111, col. 1083. 
Or llygrir yt y neb dyn yn emyl trefgord, ac na chaffo $y$ perchenhawc daly vn llwdyn arnaw, kymeret ef y creir a doet y'r tref, ac or tygant lw diarnabot, talent y rif y llwdyn ; a'r gyfreith honno a elwir "telhitor gwedy halawc $l w \|^{11}$.

(Si le blé d'un homme est abîmé à proximité d'un hameau et le propriétaire ne prend pas un animal, qu'il prenne la relique et vienne au hameau et s'ils [les habitants] prêtent un serment d'ignorance, ils paient d'après le nombre d'animaux ; et cette loi est appelée «On paie après un serment souillé».)

Malgré le fait que les habitants du hameau, interrogés par le plaignant, jurent qu'ils ne savent rien de ce qui s'est passé, il est certain que l'animal coupable est venu du hameau et, par conséquent, qu'il y a un parjure parmi ceux qui ont prêté serment. Leur serment est ainsi «souillé» et ne vaut rien.

- Gna6t ffo ar ffraeth (Fuir est l'habitude du bavard) ${ }^{12}$.

Jenny Rowland, leur éditrice la plus récente, a montré que les cycles de poèmes qui racontent les sagas de Llywarch Hen, Heledd et Urien, sont extrêmement riches en aphorismes et proverbes. Le proverbe en question fait partie d'une strophe du cycle de Llywarch Hen, dans lequel Llywarch, vieux et malin, incite son fils à faire des vœux audacieux qui deviennent de plus en plus téméraires au fur et à mesure que le dialogue entre le père et son fils progresse. Dans le contexte héroïque du poème, les vantardises de Gwên ont la valeur des serments et il meurt défendant le gué contre l'avancée des Saxons. C'est Gwên qui parle le premier :

Ny chollaf dy wyneb trin wosep wr pan wisc glew yr ystre. porthaf gnif kynn mudif lle ${ }^{13}$.

(Je ne perdrai pas ton honneur, homme prêt à la bataille / Quand les braves s'arment pour la frontière. / Je supporterai l'épreuve [du combat] avant de céder du terrain.)

11.. S. J. Williams et E. Powell, Llyfr Blegywryd, Caerdydd, Gwasg Prifysgol Cymru, 1942, p. 86.

12. J111, col. 1066.

13. Jenny Rowland, Early Welsh Saga Poetry, Cambridge, D. S. Brewer, 1990, p. 405. 
Llywarch lui rétorque en concluant ses propos sceptiques, délibérément provocants, avec le proverbe en question :

Redegawc tonn ar hyt traeth. ech adaf torrit aruaeth. kat [agdo] gnawt ffo ar ffraeth ${ }^{14}$.

(La vague court le long de la grève / une intention est vite rompue / mince protection au combat - fuir est l'habitude du bavard.)

Les proverbes traduits forment le dernier groupe de proverbes gallois selon le classement indiqué ci-dessus. Ils constituent aussi la clé de l'analyse du contexte, non des proverbes individuels, mais des recueils tout entiers. La question de leur provenance s'impose.

$*$

Une provenance celtique s'exclut aussitôt puisque aucun texte médiéval correspondant aux recueils de proverbes gallois n'est conservé en breton ni en irlandais. En plus, les recueils de proverbes en moyen anglais sont rarissimes et d'un contenu maigre à côté de ceux en gallois, bien que l'emploi des proverbes soit très répandu dans la littérature anglaise avant 1500 . Pourtant, entre les recueils gallois et français du Moyen Âge il existe un parallélisme de date et de composition qui mérite d'être exploré.

La tradition parémiologique française naît en pays anglonormand aux environs de 1150 avec la traduction du livre des Proverbes par Sanson de Nantuil et l'apparition, un peu plus tard, des Proverbia magistri Serlonis. Ensuite, vers la fin du XII siècle, un clerc inconnu à la cour de Philippe de Flandre compose les Proverbes au vilain, une pièce à proverbes qui a exercé une influence capitale sur les recueils ultérieurs. Des recueils proprement dits, il y en a 27 qui sont antérieurs au $\mathrm{XV}^{\mathrm{e}}$ siècle, renfermant chacun entre 47 et 1300 proverbes. Pour la plupart, les manuscrits qui renferment ces recueils français datent du XIII ${ }^{\mathrm{e}}$ et du XIV ${ }^{\mathrm{e}}$ siècle et ils sont ainsi contemporains des recueils gallois.

La concordance temporelle des deux traditions manuscrites laisse supposer une liaison entre l'activité parémiologique en Galles

14. Ib., p. 405. 
et celle qui fleurissait dans le milieu littéraire français, en France et en Angleterre. Cette conclusion est renforcée par les correspondances nombreuses entre les recueils au niveau du contenu. En fait, dans les recueils français et gallois médiévaux maints proverbes sont attestés dont il n'existe pas d'exemples connus en anglais ou en latin (les versions latines traduites du français mises à part). Bref, l'évidence s'accorde avec ce que l'on sait de la manière dont l'influence littéraire française s'est fait sentir en Galles au cours du Moyen Âge tardif ${ }^{15}$. Au même titre que les adaptations galloises du Pèlerinage de Charlemagne ou de La Chanson de Roland, les proverbes gallois empruntés au français (et l'idée même d'en faire des recueils) sont autant d'exemples de l'activité traductrice galloise au XIII et au $\mathrm{XIV}^{\mathrm{e}}$ siècle. Par conséquent, quand des exemples en contexte de ce dernier groupe de proverbes manquent en gallois - ce qui est souvent le cas - le parémiologue peut avancer dans son travail en contemplant le contexte des proverbes français correspondants.

$*$

Les Proverbes au vilain, un texte qui remonte à la fin du XII siècle, présentent une forme novatrice de composition proverbiale qui rompt avec la tradition parémiologique précédente ${ }^{16}$. Chaque strophe de ce recueil de proverbes en rimes est composée d'une partie narrative suivie d'un proverbe qui la glose. Si le proverbe cité à la fin de la strophe résume la leçon de la partie narrative, cette dernière éclaircit réciproquement le sens du proverbe et, puisque cette pièce à proverbes en renferme plusieurs qui sont passés en gallois mais qui ne sont pas conservés dans des textes gallois en dehors des recueils, c'est une composition indispensable afin de dégager la signification exacte des proverbes en question :

- Gorllyfnu penn y ki tra eler heibya6 $6^{17}$. Tant doit len blandi(e)r le chin que len soit passé18.

15. Voir Ceridwen Lloyd-Morgan, «L'évolution du conte gallois au Moyen Âge : tradition celtique et tradition française», dans Regards étonnés. Mélanges offerts au Professeur Gaël Milin, Brest, Les Amis de Gaël Milin, 2003, p. 213-225.

16. Sur ce texte voir E. SChUlze-BusACKeR, «Les "Proverbes au Vilain"», Proverbium 6 (1989), p. 113-127.

17. J111, col. 1064.

18. MORAWSKI, Proverbes français, op.cit., 2292. 
C'est un éloge du pragmatisme :

Cil qui son prou veut faire

De felon deputaire,

Fous est, s'il ne l'atrait.

En lui doit le suen metre

Et largement prametre,

Tant que il en ait fait

Tant doit on le chien blandir que on ait la voie passee, ce dit li vilains ${ }^{19}$.

- Caeu tin wedy brammv ${ }^{20}$.

Tart main a cul, quant pez est hors ${ }^{21}$.

Un appel à la prudence :

Maint ome ai je vëu,

Des que il ot perdu,

Qui ne voust mais jouer ;

Aussi avient souvent

Que feme se repent

De soi despuceler.

Tart est mains a cul, quant li pez est hors, ce dit li vilains ${ }^{22}$.

- Pan vynno dyn lad y gi kyndared a yrr arna $6^{23}$. Qui son chien viaut tuer la rage li met sus ${ }^{24}$.

Un exposé de la rationalité hypocrite :

Qui delivrer se veut

Dou serjant dont se deut,

Blasme li brasse et muet ;

Dit qu'il li a enblé

Quanqu'il a asenblé,

Si li tout ce qu'il puet.

19. Adolf ToBler, Li Proverbe au Vilain, Leipzig, S. Hirzel, 1895, 144.

20. J111, col. 1060.

21. Morawski, Proverbes français, op. cit., 2309.

22. ToBler, Li Proverbe au Vilain, op. cit., 261.

23. J111, col. 1081.

24. MorawsKi, Proverbes français, op. cit., 2146. 
Qui son chien veut tüer, la rage li met sus, ce dit li vilains ${ }^{25}$.

- Cos tin taea6c ef a gach yth uos ${ }^{26}$.

Gratez al vilein la coille, e il vous chiera en la palme ${ }^{27}$.

Oigniez a mastin le cul, il vous chiera en la paume ${ }^{28}$.

Claw a churl by the arse and he shits in one's hand ${ }^{29}$.

La charité vue d'un œil cynique :

Soi mëisme deçoit

Et damage i reçoit,

Qui trop felon enoure ;

Car quant plus l'aime et sert,

Damages en apert

L'en vient en petit d'oure.

Oigniez a mastin le cul, il vous chïra en la paume, ce dit li vilains ${ }^{30}$.

Bien sûr, l'évidence qui subsiste de la tradition française n'est pas toujours si obligeante et l'on arrive parfois à des impasses :

- A uo aml y uel dodet ar y iwt ${ }^{31}$.

Qui plenté a deu miel en sa pois le met ${ }^{32}$.

- Ar ny wano yn draen ny wan yn gyff ${ }^{33}$.

Qui ne point en cime ne point en racine ${ }^{34}$.

25. Tobler, Li Proverbe au Vilain, op. cit., 118.

26. J111, col. 1061.

27. Morawski, Proverbes français, op. cit., 834.

28. MORAWSKI, Proverbes français, op. cit., 1430.

29. B. J. et H. Whiting, Proverbs, Sentences and Proverbial Phrases from English Writings Mainly before 1500, Cambridge, Massachusetts, Harvard University Press, 1968, C264. Cette version anglaise du proverbe date du XVIe siècle.

30. Tobler, Li Proverbe au Vilain, op. cit., 247.

31. J111, col. 1057.

32. MoRAWSKI, Proverbes français, op. cit., 2076.

33. J111, col. 1057.

34. MoRAwsKi, Proverbes français, op. cit., 2035. 
Dans les deux cas il semble certain que le proverbe gallois est traduit du français mais, en l'absence d'un contexte concret, le sens métaphorique véritable nous échappe.

*

Ma volonté de présenter mes recherches parémiologiques à un auditoire de celtologues francophones s'explique en premier lieu par le fait qu'elles portent sur un point de jonction entre les cultures galloise et française du Moyen Âge qui est largement ignoré mais non dépourvu d'intérêt. Ensuite viennent les considérations théoriques, puisque ces recherches n'ont pas tardé à m'imposer la nécessité d'une archéologie, dans le sens foucauldien, des études celtiques afin d'examiner de près les fondements épistémologiques de la discipline.

Michel de Certeau résume ainsi («à titre d'hypothèse») la structuration, au XVIII siècle, de l'ethnologie comme discipline scientifique en antinomie à l'historiographie :

Quatre notions semblent organiser le champ scientifique dont le statut se fixe au XVIIIe siècle et qui reçoit d'Ampère son nom d'ethnologie : l'oralité (communication propre à la société sauvage, ou primitive, ou traditionnelle), la spatialité (ou tableau synchronique d'un système sans histoire), l'altérité (la différence que pose une coupure culturelle), l'inconscience (statut de phénomènes collectifs référés à une signification qui leur est étrangère et n'est donnée qu'à un savoir venu d'ailleurs)... il a également son corollaire avec l'historiographie moderne, dont la construction met en jeu, à la même époque, quatre notions opposées : l'écriture, la temporalité, l'identité et la conscience ${ }^{35}$.

Les réflexions du grand épistémologue pourraient servir comme point de départ propice pour une histoire intellectuelle des études celtiques. À l'origine, et au cours du XIX $\mathrm{X}^{\mathrm{e}}$ siècle, les études celtiques portaient tous les traits de l'ethnologie. Arnold, Renan et les grands philologues germanophones cultivaient une image généralement anhistorique des pays celtes, en amassaient des connaissances, portaient sur eux un regard d'étranger. Ce n'est que vers la fin du siècle que les études celtiques sont devenues histoire, à partir du

35. Michel de Certeau, L'écriture de l'histoire, Paris, Gallimard, 1975, p. 245-46. 
progrès du nationalisme politique dans les pays celtes. Au pays de Galles, en Bretagne, et surtout en Irlande, les celtologues de la fin $\mathrm{du} \mathrm{XIX}^{\mathrm{e}}$ siècle et du début du siècle suivant contribuaient (souvent à leur insu ou même parfois contre leur gré) à la construction et la consolidation de l'identité nationale. Pourtant la discipline n'est jamais débarrassée totalement de son héritage ethnologique et la grille épistémologique des études celtiques a encore tendance à se structurer conformément au quadrilatère ethnologique tel qu'il est décrit par de Certeau ${ }^{36}$.

Quand elle se heurte à l'évidence textuelle galloise du Moyen Âge tardif, soit cette grille épistémologique mène à des conclusions fragmentaires (voire trompeuses), soit elle s'effondre. L'analyse des recueils de proverbes en est un exemple représentatif. En les regardant à travers le prisme des études celtiques, on s'attendrait à des formules «traditionnelles», transmises à l'oral, qui reflètent le caractère gallois et ainsi de suite. Au contraire, les proverbes en question sont des textes écrits, dont l'interprétation ne saurait se limiter au milieu géographique celtique, et qui n'ont rien d'exotique puisque leurs auteurs consciemment faisaient partie intégrante de la culture européenne de l'époque.

36. Voir les exemples présentés dans Richard Glyn ROBERTS, «Achau Llafaredd», Dwned 15, 2009, p. 33-56. 
